\title{
Fetal and neonatal exposure to nicotine in Wistar rats results in increased beta cell apoptosis at birth and postnatal endocrine and metabolic changes associated with type 2 diabetes
}

Received: 5 May 2005 / Accepted: 18 July 2005 / Published online: 4 November 2005

(C) Springer-Verlag 2005

\begin{abstract}
Aims/hypothesis: Epidemiological studies report an increased risk of obesity and type 2 diabetes in children born to women who smoked during pregnancy. This study examines the effect of fetal and neonatal exposure to nicotine, the major addictive component of cigarettes, on postnatal growth, adiposity and glucose homeostasis. Methods: Female Wistar rats were given either saline (vehicle) or nicotine $\left(1 \mathrm{mg} \mathrm{kg} \mathrm{may}^{-1}\right)$ during pregnancy and lactation. Serum and pancreas tissue were collected from the infant rats at birth. Postnatal growth was assessed weekly until the rats reached 26 weeks of age and glucose homeostasis was examined by OGTTs performed at 7 and 26 weeks of age. Results: Exposure to nicotine resulted in increased postnatal growth and adiposity. Nicotine exposure also resulted in dysglycaemia at 7 and 26 weeks of age. Serum insulin concentrations were decreased in the pups exposed to nicotine at birth. This was associated with increased beta cell apoptosis (pups of saline-treated mothers $8.8 \pm 1.21 \%$ apoptotic beta cells; pups of nicotine-treated mothers $27.8 \pm 3.1 \%$ apoptotic beta
\end{abstract}

A. C. Holloway $(\bowtie) \cdot$ G. E. Lim · W. G. Foster

Department of Obstetrics and Gynecology,

McMaster University,

Rm 3N52 HSC 1200 Main Street W.,

Hamilton, ON, Canada L8N 3Z5

e-mail: hollow@mcmaster.ca

Tel.: +1-905-5259140

Fax: +1-905-5242911

J. J. Petrik

Department of Biomedical Sciences, University of Guelph,

Guelph, ON, Canada

K. M. Morrison

Department of Pediatrics, McMaster University,

Hamilton, ON, Canada

H. C. Gerstein

Department of Medicine, McMaster University,

Hamilton, ON, Canada

G. E. Lim

Department of Physiology, University of Toronto,

Toronto, ON, Canada cells). Conclusions/interpretation: Fetal and neonatal exposure to nicotine results in metabolic changes in the offspring that are consistent with obesity and type 2 diabetes. We propose that these metabolic changes may be a consequence of the initial insult to the beta cell during fetal life and that this animal model has many characteristics of diabetes in humans.

Keywords Adult-onset disease - Beta cell apoptosis · Dyslipidaemia - Glucose homeostasis $\cdot$ Nicotine .

Type 2 diabetes

Abbreviations DAPI: 4',6-Diamidino-2-Phenylindole • PND1: postnatal day 1 PND21: postnatal day 21 .

TUNEL: terminal deoxynucleotidyl transferase mediated dUTP nick end labelling

\section{Introduction}

The intrauterine environment is emerging as an important determinant of chronic adult diseases including diabetes, obesity and cardiovascular disease. A variety of physiological stresses during pregnancy are thought to contribute to the development of adult disease. One such stress is maternal cigarette smoking. Recent epidemiological studies have shown a strong relationship between maternal smoking and subsequent obesity, hypertension and type 2 diabetes in the offspring [1-8]. Although many women report a desire to quit smoking while pregnant, nicotine dependence is a significant element of most patients' smoking behaviour [9]. For this reason nicotine replacement therapy has been widely developed as a pharmacotherapy of smoking cessation, and is considered to be of benefit for pregnant women who are highly dependent and have been unable to quit smoking by other means [9-11]. However, very few studies have studied the safety of nicotine replacement during pregnancy [12].

In animal studies, prenatal exposure to nicotine alone was sufficient to produce reduced birthweight and increased body weight post-natally [13] and significantly 
higher levels of body fat in fetuses of nicotine-exposed dams at gestation day 20 (term 22 days) [14]. The fact that these are risk factors for future obesity and dysglycaemia [15] suggests that prenatal nicotine exposure may have lifelong metabolic effects that begin at birth. The effect of fetal and neonatal nicotine exposure on growth, weight, adiposity, glucose homeostasis and beta cell area is reported in this study.

\section{Subjects, materials and methods}

Maintenance and treatment of animals

All animal experiments were approved by the Animal Research Ethics Board at McMaster University, in accordance with the guidelines of the Canadian Council for Animal Care. Nulliparous 200-250-g female Wistar rats (Harlan, Indianapolis, IN, USA) were maintained under controlled lighting (12:12h light:dark) and temperature $\left(22^{\circ} \mathrm{C}\right)$ with free access to food and water. Two weeks prior to mating the dams were randomly assigned to receive either saline (vehicle) or nicotine ( $n=4$ per group). Dams were injected s.c. with $1 \mathrm{mg} \cdot \mathrm{kg}^{-1} \cdot \mathrm{day}^{-1}$ nicotine bitartrate (Sigma Aldrich, St Louis, MO, USA) or saline for 14 days prior to mating, and during pregnancy until weaning. Pups were weighed after birth (postnatal day 1; PND1) and the litters were culled to eight at birth, retaining males in preference to assure uniformity of litter size between treated and control litters. Trunk blood was collected from culled animals and allowed to clot at $4{ }^{\circ} \mathrm{C}$, then centrifuged and stored at $-80^{\circ} \mathrm{C}$ until analysis for insulin and glucose concentrations. Serum glucose concentrations were measured using a commercially available kit for the glucose oxidase method (Pointe Scientific, Canton, MI, USA), and insulin levels were measured on an ultra-sensitive rat insulin ELISA (Crystal Chem, Downers Grove, IL, USA). The insulin ELISA kit had a detection limit of $5 \mathrm{pg} / \mathrm{ml}$ with intra- and interassay variabilities of 5.5 and $4.8 \%$, respectively. After weaning at postnatal day 21 (PND21), male offspring were selected randomly and caged as sibling pairs. Pups were weighed weekly from PND1 until 26 weeks of age. Fasting serum samples were collected at 7, 15 and 26 weeks and were analysed for triglyceride concentrations using a commercially available assay (Pointe Scientific).

\section{Glucose tolerance tests}

Glucose tolerance was serially investigated in nicotineexposed and control rats at 7 and 26 weeks $(n=16$ per group). Serum concentrations of insulin and glucose were measured in saphenous vein samples obtained after food deprivation at 0 (0900 hours), 30 and $120 \mathrm{~min}$ after rats were given $2 \mathrm{~g} / \mathrm{kg}$ glucose (Sigma-Aldrich) in water by gavage. Blood samples were allowed to clot at $4^{\circ} \mathrm{C}$, then centrifuged and stored at $-80^{\circ} \mathrm{C}$ until used for measurement of serum glucose and insulin concentrations as described above.
Immunohistochemistry: beta cell area

To assess whether nicotine exposure can alter pancreas development, pancreas tissue was removed from all culled pups at birth (PND1). The pancreas from each animal was weighed and then fixed by immersion in $10 \%$ neutralbuffered formalin (EM Science, Gibbstown, NJ, USA) at $4^{\circ} \mathrm{C}$ overnight, washed in PBS and embedded in paraffin. Immunohistochemical detection of insulin was performed on 5- $\mu \mathrm{m}$ sections of neonatal pancreatic tissue (PND1; $n=10$ per group). Tissue sections were deparaffinised in xylene, rehydrated and washed in PBS. After quenching in methanol of endogenous peroxidase activity, antigen retrieval was performed in $10 \mathrm{mmol} / \mathrm{l}$ citrate buffer $(\mathrm{pH}$ 3.0 ) followed by incubation with $10 \%$ normal goat serum and $1 \%$ BSA. Sections were then incubated overnight at $4^{\circ} \mathrm{C}$ with the primary antibody, a polyclonal, guinea-pig anti-human insulin antibody (1:150 dilution) (DakoCytomation, Carpinteria, CA, USA) which has been shown by the manufacturer to cross-react with rat insulin peptides. Sections were then washed in PBS, and immunostaining was identified using the Vectastain kit (Vector Laboratories, Burlinghame, CA, USA) with diaminobenzadine as the chromogen. Tissue sections were counterstained, dehydrated and mounted with Permount (Fisher Scientific, Fair Lawn, NJ, USA). Control sections were incubated with $1 \%$ BSA in PBS in place of the primary antibody. All measurements were performed at $10 \times$ magnification, and the whole pancreas was analysed. Immunopositive cells were identified and the beta cell area was calculated as a percentage of the total area of the section using integrated morphometry software (METAMORPH, Universal Imaging, Downington PA, USA). At least 25 fields were counted per animal.

Immunohistochemistry: detection of beta cell apoptosis

Histological sections of pancreas from animals at PND1 were processed and fixed as described above. Apoptotic islet cells were detected using a terminal deoxynucleotidyl transferase mediated dUTP nick end labelling (TUNEL) kit (Roche Applied Science, Laval, PQ, Canada) following the manufacturer's protocol. Briefly, after fixation, tissues and cells were made permeable in $0.1 \%$ Triton X-100 for $15 \mathrm{~min}$, washed in PBS, and incubated with the fluorescein isothiocyanate-conjugated TUNEL enzyme for $60 \mathrm{~min}$ to detect DNA fragmentation. Nuclei were counterstained with propidium iodide and images were obtained on an Olympus BX-61 microscope. For analysis, ten fields of view at $250 \times$ magnification were counted for each animal ( $n=5$ animals per group). To evaluate the percentage of beta cells that were apoptotic in the pancreas islets, pancreas tissue from pups at PND1 was processed for double-label immunofluorescence. For double-label immunofluorescence, sections were initially analysed on an apoptosis detection kit, as described above. Following staining for fragmented DNA, sections were incubated overnight at 
Fig. 1 Postnatal growth of the offspring of dams given saline (control: closed circles, $n=16$ ) or nicotine bitartrate $(1 \mathrm{mg} / \mathrm{kg} /$ day; open circles, $n=16$ ) during pregnancy and lactation. Data are presented as mean \pm SEM. Offspring of nicotine-exposed dams were significantly heavier than those of saline-treated controls at the end of the study (26 weeks of age, $p<0.01$ ). The total growth response (AUC) of the pups exposed to nicotine in utero and during lactation was significantly $(p<0.001)$ higher than the saline-exposed group (AUC control pups: 9,299 231.4; nicotine-exposed pups: $10,826 \pm 121.2)$

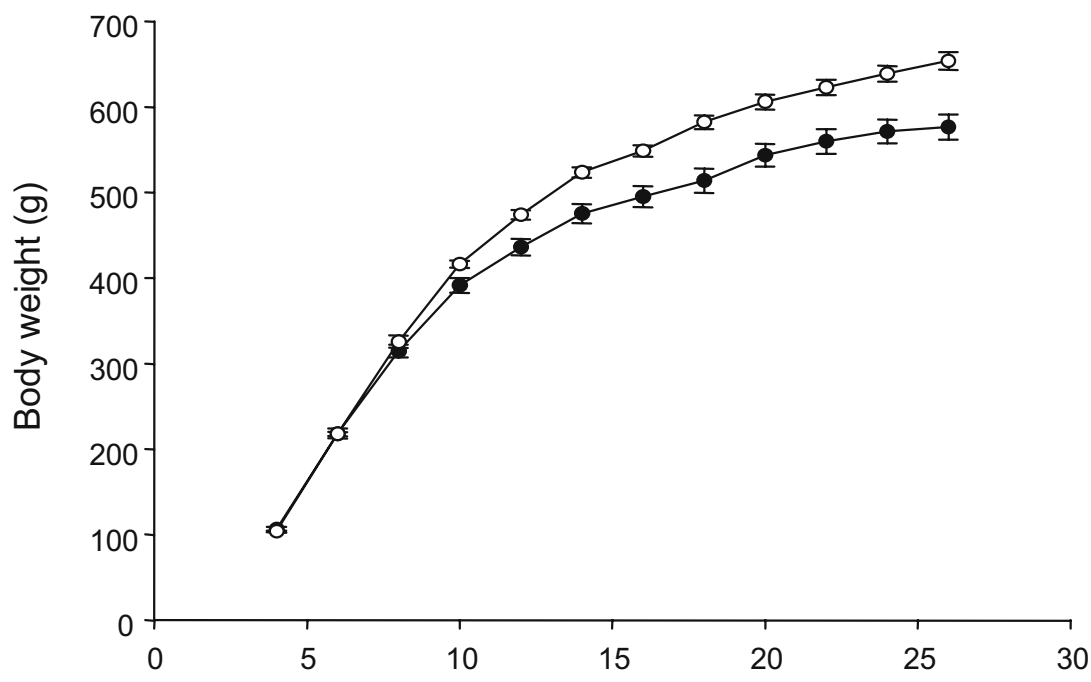

Age (weeks)

$4{ }^{\circ} \mathrm{C}$ with an anti-insulin antibody (DakoCytomation, Carpinteria, CA, USA; 1:150). A Texas Red-conjugated antimouse antibody (Vector Laboratories, Burlington, ON, USA; 1:100 dilution) was used for detection. Nuclei were counterstained with propidium iodide or DAPI and images of the specimens were obtained on an Olympus BX-61 fluorescent microscope.

\section{Statistical analysis}

All statistical analyses were performed using SigmaStat (v.2.03, SPSS, Chicago, IL, USA), and $t$-test or analyses of variance (ANOVA) followed by appropriate post-hoc tests when significance was indicated by the ANOVA $(\alpha=0.05)$. Data were tested for normality as well as equal variance, and when normality or variance tests failed, data were analysed using Mann-Whitney rank sum test or KruskalWallis one-way ANOVA on ranks. Area under the curve for the total glucose response following the glucose tolerance test and postnatal growth was assessed using the trapezoidal rule.

\section{Results}

Birth phenotype

Birthweight was reduced in rats that were exposed to nicotine in utero (saline $6.54 \pm 0.099 \mathrm{~g}$, nicotine $6.29 \pm 0.056 \mathrm{~g}$, $n=47$ and $59, p<0.05$ ) whereas the litter size, sex ratio and gestational length were unaffected (data not shown).

Postnatal growth and adiposity

There was no significant effect of treatment on weight gain during lactation and no significant difference in body weight at weaning. Following weaning the growth trajectory of the offspring of the nicotine-treated dams was significantly enhanced, resulting in increased body weight at 26 weeks of age compared to control offspring (Fig. 1). We have previously shown that this weight gain is partly the result of a significant increase in body fat at 26 weeks in nicotine-exposed pups [16]. Furthermore, in this study hypertriglyceridaemia was evident in nicotine-exposed pups at 7 weeks of age (control pups $0.81 \pm 0.119 \mathrm{mmol} / \mathrm{l}$, nicotine-exposed pups $1.08 \pm 0.071 \mathrm{mmol} / 1, p=0.052, n=16$ per group) and persisted until 26 weeks of age (control pups $1.69 \pm 0.063 \mathrm{mmol} / \mathrm{l}$, nicotine-exposed pups $2.00 \pm$ $0.19 \mathrm{mmol} / 1, p=0.044, n=16$ per group).

\section{Glucose homeostasis}

Serum glucose and insulin levels were measured at PND1 and at 7 and 26 weeks of age following an overnight fast and following an oral glucose challenge at 7 and 26 weeks of age. Fetal nicotine exposure did not alter basal serum glucose concentrations at birth but caused a significant reduction in basal serum insulin concentrations in the nicotine-exposed offspring at PND1 (Table 1). At 7 weeks of age, there was no difference in fasting serum glucose or insulin concentrations; however, nicotine-exposed off-

Table 1 Effect of in utero exposure to nicotine on serum hormone concentrations, pancreatic beta cell area and islet apoptosis at PND1

\begin{tabular}{lclc}
\hline PND1 & Saline & Nicotine & $p$ value \\
\hline Glucose $(\mathrm{mmol} / \mathrm{l})$ & $0.82 \pm 0.094$ & $0.89 \pm 0.081$ & 0.59 \\
Insulin $(\mathrm{ng} / \mathrm{ml})$ & $0.36 \pm 0.048$ & $0.23 \pm 0.032 *$ & 0.04 \\
Beta cell area (\% of total) & $2.5 \pm 0.29$ & $3.0 \pm 0.31$ & 0.23 \\
Islet apoptosis (\% of total) & $4.2 \pm 0.09$ & $8.7 \pm 0.61 *$ & $<0.001$ \\
\hline
\end{tabular}

Data are presented as mean $\pm \mathrm{SEM}$ 
spring exhibited dysglycaemia following administration of the oral glucose challenge with no significant difference in the insulin response to the glucose challenge between the two groups of animals (Fig. 2).
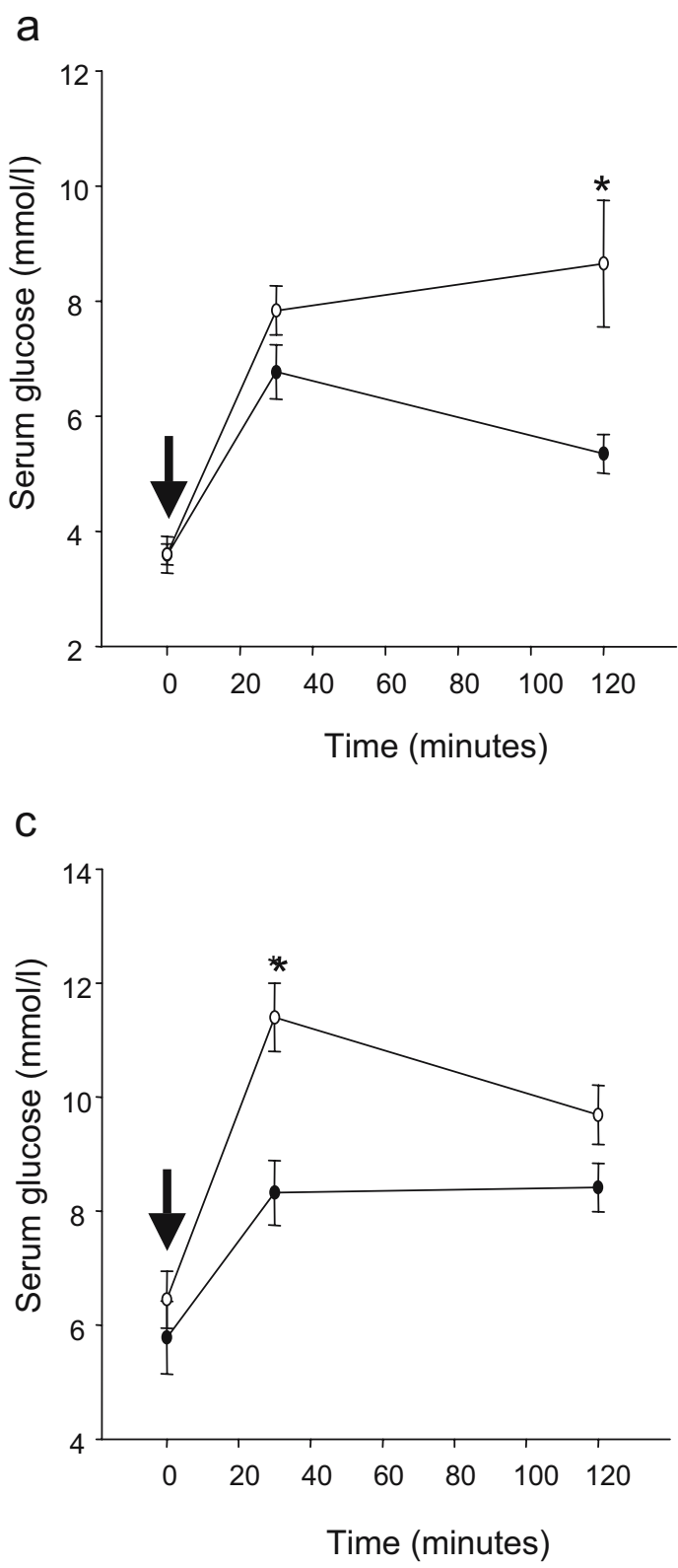

Fig. 2 Serum glucose and insulin concentrations following administration of an oral glucose load $(2 \mathrm{~g} / \mathrm{kg}$ body weight represented by the arrow) at 7 and 26 weeks of age for the offspring of dams given saline (control: closed circles, $n=16$ ) or nicotine bitartrate, $(1 \mathrm{mg} / \mathrm{kg} / \mathrm{day}$; open circles, $n=16)$ during pregnancy and lactation. Data are presented as mean \pm SEM. $* p<0.05$ for difference from control animals at the same time point. Serum glucose concentrations at 7 weeks of age (a). Nicotineexposed rats had higher blood glucose levels at $120 \mathrm{~min}$ postchallenge $(p<0.01)$ than controls. Although the serum glucose concentration in nicotine-exposed offspring was higher at 30-min post-challenge, this did not reach significance $(p=0.07)$. The total glucose response (AUC) following glucose administration was significantly elevated in the nicotine-exposed offspring (AUC control pups 12,621 \pm 762.9 ; AUC nicotine-exposed pups 16,376 \pm 985.5, $p=0.006$ ) compared to saline controls. b Serum insulin
At 26 weeks there was no significant difference in basal fasting serum glucose levels between saline- and nicotineexposed offspring, but the fasting insulin concentrations were significantly elevated in the nicotine-exposed off-

b
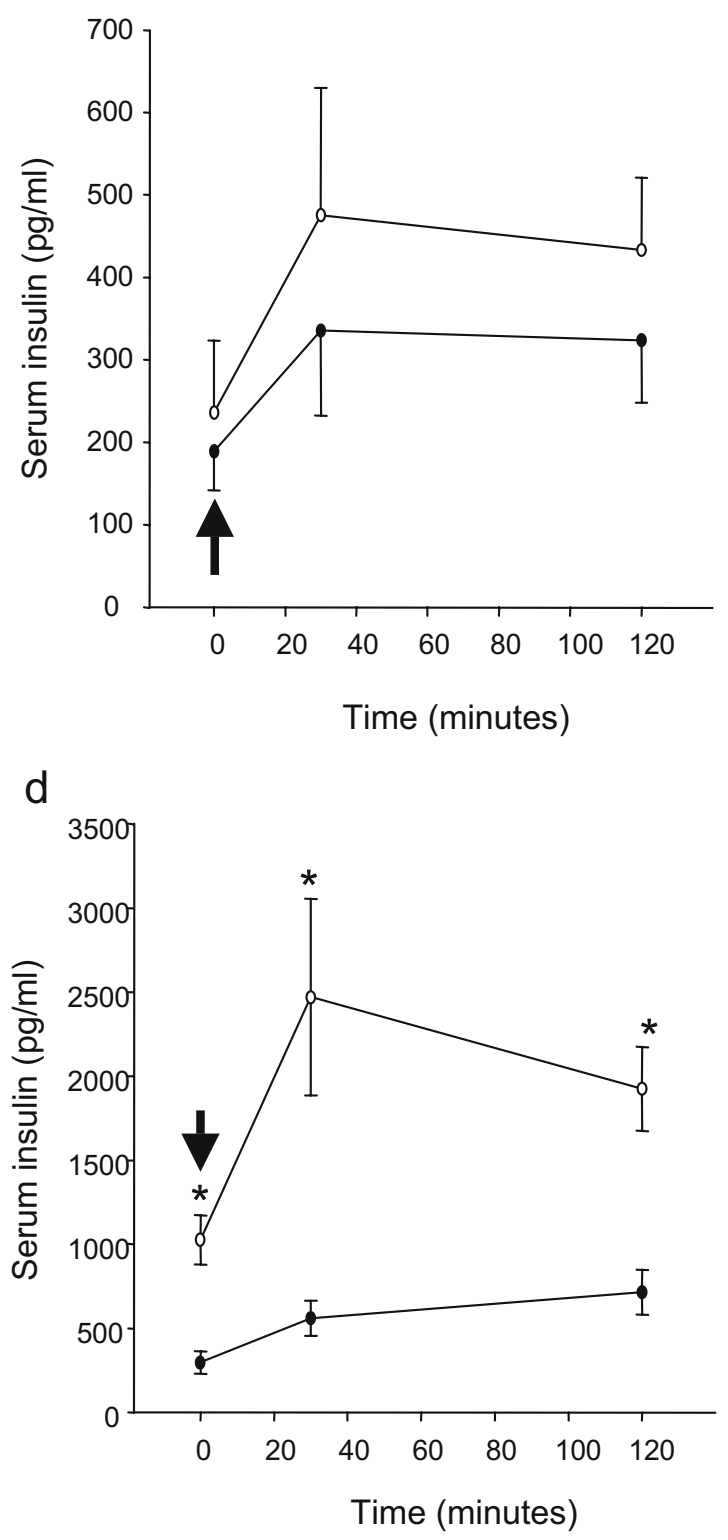

concentrations at 7 weeks of age. There was no significant difference at any time point in the total insulin response (AUC) between the two groups. c Serum glucose concentrations at 26 weeks of age. Nicotine-exposed rats had higher $(p=0.001)$ peak glucose concentrations at $30 \mathrm{~min}$. Although the serum glucose concentrations at 120-min post-challenge were higher than those of the saline controls, this did not reach significance $(p=0.07)$. The total glucose response to the challenge (AUC) was higher in the nicotineexposed animals than in saline controls (AUC control rats 17,341. 1073.5, $n=16$; AUC nicotine-exposed rats 21,890 $\pm 927.8, n=16$; $p=0.004$ ). d Serum insulin concentrations following an OGTT at 26 weeks of age. Nicotine-exposed offspring had higher serum insulin concentrations at all time points $(p<0.01$ for all). The total insulin response to the glucose challenge was higher in the offspring of nicotine-treated dams (AUC control rats $77.3 \pm 10.5$; AUC nicotine-exposed rats $250.3 \pm 42.6 ; p<0.001)$ 
spring. Nicotine-exposed rats exhibited impaired glucose homeostasis and a significantly higher insulin response to the glucose challenge (Fig. 2).

\section{Beta cell area and apoptosis}

To assess whether the differences in serum insulin levels at PND1 were a result of an altered number of beta cells or increased beta cell death, we evaluated both the beta cell area and the percentage of apoptotic beta cells in the pancreas at PND1. There was no significant effect of treatment on beta cell area; however, the offspring of the nicotine-exposed dams had significantly higher $(p<0.001)$ levels of apoptosis in the pancreatic islet cells compared to the offspring of the saline-exposed dams (Table 1). To confirm that the apoptotic islet cells were in fact insulinsecreting cells, pancreatic tissue from animals at PND1 was dual-labelled with insulin and TUNEL staining. In the pancreas tissue from control offspring there were $8.8 \pm$ $1.21 \%$ TUNEL/insulin-positive beta cells and in the nicotine-exposed offspring there were $27.8 \pm 3.1 \%$ TUNEL/ insulin-positive beta cells $(p<0.001)$.

\section{Discussion}

Recent epidemiological studies have shown that the offspring of women who smoke during pregnancy have an increased risk of developing obesity, hypertension and type 2 diabetes [1-8]. The mechanism(s) underlying these associations are unknown. The results from this study demonstrate for the first time that nicotine exposure alone, at concentrations which are representative of human exposure $[17,18]$, during pregnancy and lactation results in endocrine and metabolic changes in the offspring that are consistent with the disturbed glucose metabolism that may lead to type 2 diabetes.

In the present study we have shown that fetal and neonatal exposure to nicotine results in impaired glucose homeostasis in response to a glucose challenge prior to a measurable difference in body weight (7 weeks of age), suggesting that in this model the metabolic changes associated with the onset of type 2 diabetes precede obesity. Furthermore, we found that nicotine exposure during the fetal period alone resulted in a significant reduction in serum insulin concentrations at PND1 in association with a significant increase in the number of apoptotic islet cells. We therefore propose that the nicotine-induced damage to the beta cell during fetal development results in permanent changes in pancreatic function, which are evident as impaired glycaemic control at 7 weeks of age and which persist until adulthood (26 weeks of age).

Similarly, other in utero insults including glucocorticoid administration, uteroplacental insufficiency and fetal undernutrition have been shown to result in long-lasting functional deficiencies in the endocrine pancreas [19-22]. At 26 weeks of age nicotine-exposed offspring exhibit dysglycaemia and increased serum insulin concentrations.
The elevated insulin concentrations at 26 weeks of age are probably a result of the dysglycaemia and/or impaired sensitivity to insulin, in a similar manner to glucose intolerance in humans. Because dysglycaemia precedes obesity in this model, we speculate that the metabolic changes associated with type 2 diabetes are not caused by obesity, but that this initial underlying beta cell defect could represent a common antecedent for the postnatal obesity and the dysglycaemia observed by us.

We propose that nicotine acts directly at the fetal beta cell to cause apoptosis, possibly by increasing oxidative stress at that location. In adult rats, nicotine exposure has been shown to produce oxidative tissue injuries in vivo [23, 24] and can induce oxidative stress in pancreatic tissue in vitro [25]. Oxidative stress has been shown to decrease insulin secretion and to increase apoptosis in beta cells [reviewed in Ref. 26], results which are reflected by the decrease in serum insulin concentrations and increased beta cell apoptosis observed at PND1 in this study. Alternatively nicotine may act directly at the nicotinic receptors on the beta cell to inhibit insulin secretion, as has been demonstrated in isolated rat islets in vitro [27]. Further studies will be required to address this question and to determine the specific developmental stages that are sensitive to nicotine exposure.

Results from this study have shown that (1) nicotine, at levels that are relevant for human exposure, results in postnatal obesity and impaired glucose homeostasis and (2) that these postnatal changes may be a result of an underlying defect in the pancreatic beta cell. On the basis of our results, we propose that the long-term postnatal health consequences of nicotine exposure warrant further investigation. Our results also raise concerns regarding the safety of nicotine replacement therapy during pregnancy and lactation.

Acknowledgements This work was supported by an operating grant from the Canadian Institutes of Health Research in Nutrition, Metabolism and Diabetes to A. C. Holloway and K. M. Morrison (MOP 69025), and a fellowship from the Heart and Stroke Foundation to K. M. Morrison. H. C. Gerstein holds the Population Health Institute Chair in Diabetes Research (sponsored by Aventis). We thank Ms Sandra Stals, Ms Lisa Kellenberger and the staff of the McMaster University Central Animal Facility for their excellent technical assistance.

\section{References}

1. Bergmann KE, Bergmann RL, von Kries R et al (2003) Early determinants of childhood overweight and adiposity in a birth cohort study: role of breast feeding. Int J Obesity 27:162-172

2. Blake KV, Currin LC, Evans SF et al (2000) Maternal cigarette smoking during pregnancy, low birth weight and subsequent blood pressure in early childhood. Early Hum Dev 57:137-147

3. Montgomery SM, Ekbom A (2002) Smoking during pregnancy and diabetes mellitus in a British longitudinal birth cohort. BMJ 324:26-27

4. Morley R, Leeson Payne C, Lister G, Lucas A (1995) Maternal smoking and blood pressure in 7.5 to 8 -year old offspring. Arch Dis Child 72:120-124 
5. Toschke AM, Koletzko B, Slikker W Jr, Hermann M, von Kries $\mathrm{R}$ (2002) Childhood obesity is associated with maternal smoking in pregnancy. Eur J Pediatr 161:445-448

6. Vik T, Jacobsen G, Vatten L, Bakketeig LS (1996) Pre- and postnatal growth in children of women who smoked in pregnancy. Early Hum Dev 45:245-255

7. von Kries R, Toschke AM, Koletzko B, Slikker W Jr (2002) Maternal smoking during pregnancy and childhood obesity. Am J Epidemiol 156:954-961

8. Wideroe M, Vik T, Jacobsen G, Bakketeig LS (2003) Does maternal smoking during pregnancy cause childhood overweight? Paediatr Perinat Epidemiol 17:171-179

9. Okuyemi KS, Ahluwalia JS, Harris KJ (2000) Pharmacotherapy of smoking cessation. Arch Fam Med 9:270-281

10. Ontario Medical Association (1999) Rethinking stop-smoking medications: myths and facts. Available at http://www.oma.org/ phealth/stopsmoke.htm

11. Peters MJ, Morgan LC (2002) The pharmacotherapy of smoking cessation. Med J Aust 176:486-490

12. Oncken CA, Kranzler HR (2003) Pharmacoptherapies to enhance smoking cessation during pregnancy. Drug Alcohol Rev 22:191-202

13. Newman MB, Shytle RD, Sanberg PR (1999) Locomotor behavioural effects of prenatal and postnatal nicotine exposure in rat offspring. Behav Pharmacol 10:699-706

14. Williams CM, Kanagasabai T (1984) Maternal adipose tissue response to nicotine administration in the pregnant rat: effects on fetal body fat and cellularity. Br J Nutr 51:7-13

15. Ravussin E, Smith SR (2002) Increased fat intake, impaired fat oxidation, and failure of fat cell proliferation result in ectopic fat storage, insulin resistance, and type 2 diabetes mellitus. Ann NY Acad Sci 967:363-378

16. Gao YJ, Holloway AC, Zeng ZH et al (2005) Prenatal exposure to nicotine causes postnatal obesity and altered perivascular adipose tissue function. Obesity Res 13:687-692
17. Micu AL, Miksys S, Sellers EM, Koop DR, Tyndale RF (2003) Rat hepatic CYP2E1 is induced by very low nicotine doses: an investigation of induction, time course, dose response, and mechanism. J Pharmacol Exp Ther 306:941-947

18. de Weerd S, Thomas CM, Kuster JE, Cikot RJ, Steegers EA (2002) Variation of serum and urine cotinine in passive and active smokers and applicability in preconceptional smoking cessation counselling. Environ Res 90:119-124

19. Holness MJ, Langdown ML, Sugden MC (2000) Early-life programming of susceptibility to dysregulation of glucose metabolism and the development of type 2 diabetes mellitus. Biochem J 349:657-665

20. Petrik J, Reusens B, Arany E et al (1999) A low protein diet alters the balance of islet cell replication and apoptosis in the fetal and neonatal rat and is associated with a reduced pancreatic expression of insulin-like growth factor-II. Endocrinology 140:4861-4873

21. Shen CN, Seckl JR, Slack JM, Tosh D (2003) Glucocorticoids suppress beta-cell development and induce hepatic metaplasia in embryonic pancreas. Biochem J 375:41-50

22. Simmons RA, Templeton LJ, Gertz SJ (2001) Intrauterine growth retardation leads to development of type 2 diabetes in the rat. Diabetes 50:2279-2286

23. Husain K, Scott BR, Reddy SK, Somani SM (2001) Chronic ethanol and nicotine interaction on rat tissue antioxidant defense system. Alcohol 25:89-97

24. Yildiz D (2004) Nicotine, its metabolism and an overview of its biological effects. Toxicon 43:619-632

25. Wetscher GJ, Bagchi M, Bagchi D et al (1995) Free radical production in nicotine treated pancreatic tissue. Free Radic Biol Med 18:877-882

26. Kajimoto Y, Kaneto H (2004) Role of oxidative stress in pancreatic $\beta$-cell dysfunction. Ann NY Acad Sci 1011:168-176

27. Yoshikawa H, Hellstrom-Lindahl E, Grill V (2005) Evidence for functional nicotinic receptors on pancreatic $\beta$ cells. Metabolism 54:247-254 